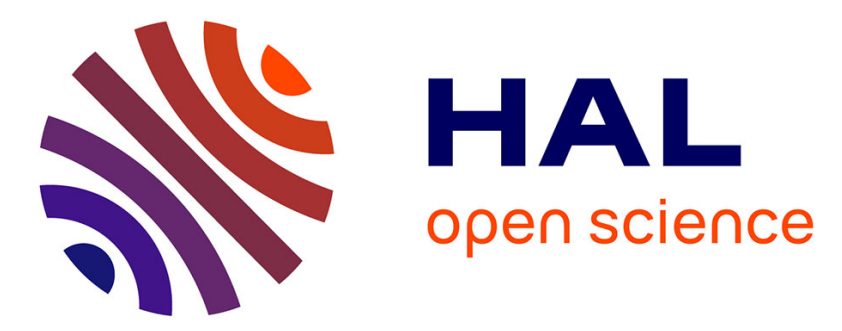

\title{
Micro-computed tomography for discriminating between different forming techniques in ancient pottery: new segmentation method and pore distribution recognition
}

\author{
Vanna Lisa Coli, Louise Gomart, Didier F Pisani, Serge X. Cohen, Laure
} Blanc-Féraud, Juliette Leblond, Didier Binder

\section{To cite this version:}

Vanna Lisa Coli, Louise Gomart, Didier F Pisani, Serge X. Cohen, Laure Blanc-Féraud, et al.. Microcomputed tomography for discriminating between different forming techniques in ancient pottery: new segmentation method and pore distribution recognition. Archaeometry, 2021, 10.1111/arcm.12693 . hal-03225382v2

\author{
HAL Id: hal-03225382 \\ https://hal.science/hal-03225382v2
}

Submitted on 17 May 2021

HAL is a multi-disciplinary open access archive for the deposit and dissemination of scientific research documents, whether they are published or not. The documents may come from teaching and research institutions in France or abroad, or from public or private research centers.
L'archive ouverte pluridisciplinaire HAL, est destinée au dépôt et à la diffusion de documents scientifiques de niveau recherche, publiés ou non, émanant des établissements d'enseignement et de recherche français ou étrangers, des laboratoires publics ou privés.

$$
\text { Copyright }
$$




\title{
Micro-computed tomography for discriminating between different forming
} techniques in ancient pottery: new segmentation method and pore distribution recognition

\author{
V.L. Coli ${ }^{1 *}$, L. Gomart ${ }^{2}$ D.F. Pisani, ${ }^{3}$ S. Cohen, \\ L. Blanc-Féraud, ${ }^{5}$ J. Leblond, $\quad$ D. Binder ${ }^{1}$
}

May 17, 2021

\begin{abstract}
Micro-computed tomography is a valuable tool for studying ancient ceramics technology. Analysing pottery 3-D images is a challenging issue, the data being extremely noisy and heterogeneous.

Quantitative criteria are introduced for the characterisation of a previously unrecognised pottery building method, the Spiralled Patchwork Technology $(\mathrm{SPT})$.

An analytical protocol has been implemented which applies to 3-D reconstructions of ceramic sherds and integrates automatic segmentation of porous systems and shape recognition using Hough transform. It enables discriminating between SPT and other techniques for vessels manufacture, and opens up many perspectives for independent characterisation of ancient technical gestures in ceramic technology.

\footnotetext{
${ }^{1}$ Université Côte d'Azur, CNRS, CEPAM, 06300 Nice, France

*Corresponding author, e-mail: Vannalisa.Coli@cepam.cnrs.fr

${ }^{2}$ Université Paris 1 Panthéon-Sorbonne, CNRS-UMR 8215 Trajectoires, 92023 Nanterre, France

${ }^{3}$ Université Côte d'Azur, CNRS, LP2M, Nice, France

${ }^{4}$ IPANEMA USR3461, CNRS, Université Paris-Saclay, Ministère de la Culture, UVSQ, MNHN, 91192 Saint-Aubin, France

${ }^{5}$ Université Côte d'Azur, CNRS, Inria, Laboratoire I3S UMR 7271, Sophia Antipolis, France

${ }^{6}$ INRIA, Team Factas, B.P. 93, 06902 Sophia Antipolis Cedex, France
} 


\section{Introduction}

Applying micro-computed tomography $(\mu-\mathrm{CT})$ on ancient pottery technology presents specific challenges related to the nature of the clay material itself, which is highly heterogeneous from mineralogical, textural and chemical viewpoints, presents varying degrees of alteration and, once fired, different microstructures according to the firing technology. While several recent studies have demonstrated the great potential of $\mu$-CT to visualise the internal structure of ancient pottery [Gomart et al. 2017, Kahl and Ramminger 2012, Kozatsas et al. 2018, Park et al. 2019, Sanger et al. 2013, Sanger 2016, Sanger 2017], using this method for the characterisation of ancient forming techniques remains arduous. To assess ancient pottery forming techniques via $\mu$-CT, it is indeed necessary to have access to the internal architecture of large portions of the vessels or of entire vessels, which implies the acquisition of large volume samples and data processing on large regions of interest (ROI). The heterogeneity of ceramics' internal structure making data segmentation and analysis on large ROIs particularly complex, there is, to the Authors' knowledge, no study focused on the forming of ceramics that has gone beyond the analysis of tomographic data in 2-D (virtual slices) or the sole qualitative analysis of 3-D data. In the context of the first Neolithic of the north-western Mediterranean, a previously unrecognised ceramic fashioning sequence was recently discovered, which (1) differs from all the technical sequences identified so far in ancient agricultural contexts in Europe and (2) has not been recognised in any other archaeological or modern context [Gomart et al. 2017]. The analysed vessels were entirely constructed by juxtaposition of circular patches of regular diameter $(\approx 45 \mathrm{~mm}$ ) previously formed using a spiralled coil (see Fig. 1 ), in stark contrast with the coiling technique (superimposition of long rolls of clay) that dominates in Early European Neolithic contexts (e.g. [Commenge 2009, Gomart 2010, Gomart 2014, Kreiter et al. 2017, Salanova et al. 2010]).

This new operational sequence was identified by means of macro- and microscopic analyses, based on the thorough examination of (1) the spatial organisation of pores and mineral inclusions, as well as the associated discontinuities in radial and equatorial sections; (2) the characteristics of the surface topography; (3) the variations in wall thickness and texture, and (4) the fracture networks. The diagnostic macro- and microtraces for this technical sequence are as follows: uneven surface topography, networks of curvilinear fractures and uneven topography on the surfaces, circular 
organisation of the pores and inclusions; curvilinear discontinuities in the tangential plane; circular configurations associated with long oblique discontinuities in the radial plane. As no source of comparison was available for this operational sequence, a first set of $\mu$-CT data was acquired in order to create a body of evidence independent from the surface reading. This offered 2-D and 3-D images that were visually in line with the macroscopic observations, i.e. circular and curvilinear alignments of pores and mineral inclusions [Gomart et al. 2017]. The goal of this research is to go beyond the simple visualisation of the tomographic images obtained by introducing quantitative criteria in 3-D on a larger series of $\mu$-CT data in order to build an unbiased body of evidence for the spiralled patchwork technology (SPT) and discriminate it from the regular coiling technique.

\section{Materials and methods}

Pottery is characterised by high intrinsic porosity whose profile varies according to the clay material used [Coletti et al. 2016, Drieu et al. 2019]). In addition to this intrinsic porosity, there is also a porosity linked to the technical gestures implemented on the paste during the forming of the pottery. Several experimental studies have shown that (1) the different types of pressure applied to the plastic clay orient the pores according to specific patterns (e.g. [Thér 2016]); (2) the assembling of clay elements during forming traps air pockets in the paste, creating specific discontinuities in the internal structure of the pottery, whose shape varies according to the fashioning technique used (e.g. [Livingstone Smith 2001]).

The spatial organisation of the mineral inclusions also characterises the internal structure of a pottery and is subjected to the type of pressure applied during forming. Thus, mapping the pores and these inclusions provides a reproducible indication of sequences of technical gestures [Pierret et al. 1996].

The mineral inclusions include a large variety of heterogeneous materials, which can differ from one vessel to another (according to the type of clayey earth used) or even inside the same vessel (some earths being more heterogeneous than others), while the pores correspond to more homogeneous regions (see Fig. 1, central column). As a result, the pores are more easily identifiable and segmentable than mineral inclusions, which often display various intensity values corresponding to different absorption properties. In order to make the new protocol applicable to the 
whole sample set, as well as other pottery assemblages characterised by different clay materials, the focus of the analysis is on pore distributions.

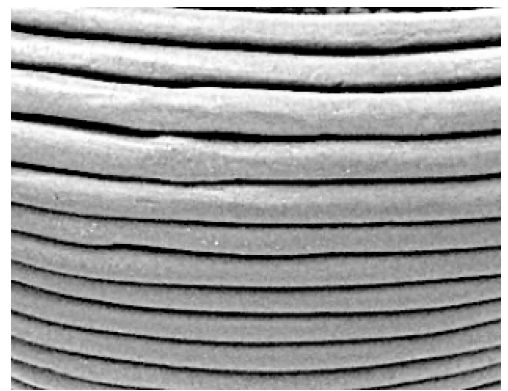

(a)

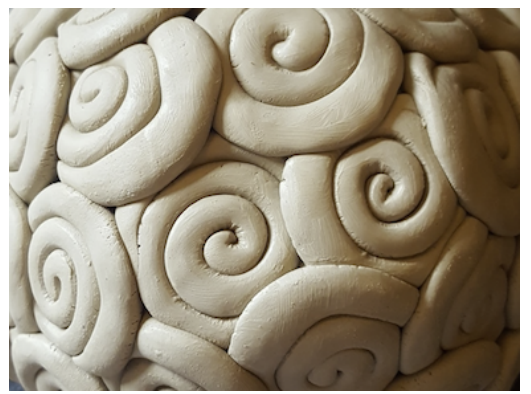

(d)

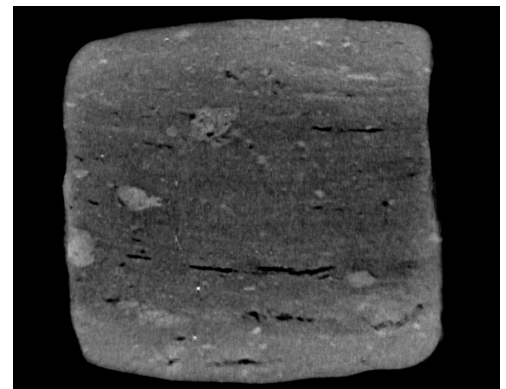

(b)

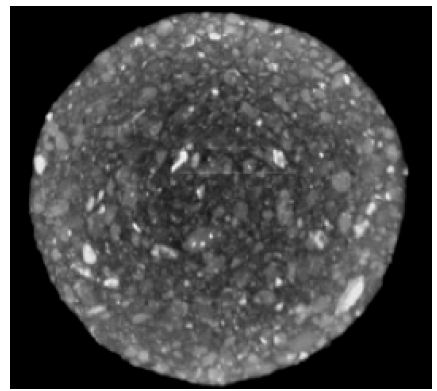

(e)

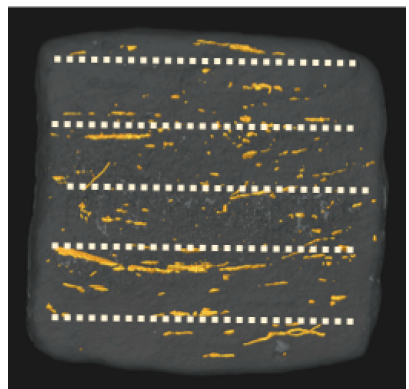

(c)

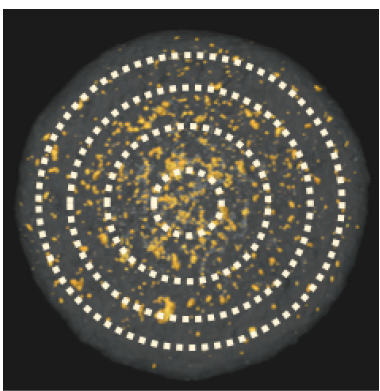

(f)

Figure 1: Scheme for considered pottery building techniques. First row: coiling technique, second row: Spiralled Patchwork Technology. (a) and (d): experimental vase; (b) and (e): tomographic image of a slice issued from an experimental control; (c) and (f): distribution of pores (in yellow) inside the experimental control's volume. (b)-(c): central slice from sherd C1, $6 \mathrm{~cm}$ side approximately. (e)-(f): central slice from sherd SP1, $5 \mathrm{~cm} \varnothing$ approximately.

\subsection{Selection of the samples}

A study was conducted on archaeological fragments showing clear diagnostic macrotraces (e.g. for the SPT, networks of curvilinear fractures and uneven topography on the surfaces) and ceramic controls, i.e. ceramic sherds produced in controlled conditions using (1) on the one hand the newly identified SPT, and (2) on the other hand the regular coiling technique. These control samples were produced using clayey earths mineralogically and texturally analogous to that used to produce the archaeological ceramics here analysed, as defined on the basis of a petrographic approach. The 6 archaeological fragments are issued from the Castellar

- Pendimoun rock shelter (Impresso-cardial Neolithic; Early 6th millennium BCE, [Binder et al. 1993, Binder et al. 2020, Drieu et al. 2020, Gomart et al. 2017]) and from Agay - La Cabre settlement (Late Southern Chassey Culture, Early 4th millennium 
BCE, [unpublished]), both located close to the Mediterranean coast in south-eastern France. The corpus of 6 archaeological samples is completed by 10 experimental controls, 3 issued from the coiling technique and 7 issued from the SPT, and it is listed in Table 1. As the archaeological SPT sherds could be made by a part of either a single patch or several overlapping patches, 6 single-patch experimental controls and 1 double-patched experimental control are considered as well.

\begin{tabular}{ccccc}
\hline \multicolumn{2}{c}{ Coiling technique } & & \multicolumn{2}{c}{ SPT } \\
\cline { 1 - 1 } Archaeological & Exp. control & & Archaeological & Exp. control \\
\hline AC1 (V1.9) & C1 (ControleCoil) & & ASP1 (AP0013 sherd1) & SP1 (ControleSPT) \\
& C2 (V3.2) & & ASP2 (AP27928, V1.1) & SP2 (DoubleSPT) \\
& C3 (V4.8) & & ASP3 (AP0013 sherd2, V3.6) & SP3 (V1.3) \\
& & ASP4 (V4.1) & SP4 (V1.4) \\
& & & ASP5 (AP47643, V4.2) & SP5 (V1.8) \\
& & & SP6 (V2.1) \\
& & & SP7 (V2.2) \\
\hline
\end{tabular}

Table 1: List of archaeological sherds and experimental controls.

The samples were scanned using a SkyScan-1178 X-ray $\mu$-CT system (Bruker) with two $1280 \times 1024$ X-ray cameras CCD1300D1, beam energy of $60 \mathrm{kV}$, a $0.5 \mathrm{~mm}$ thick aluminium filter, slow scan mode, $0.7^{\circ}$ rotation, $420 \mathrm{~ms}$ and no binning applied. The software used for reconstruction is NRecon 1.6.6.0, ring artifact correction was applied with parameter 12, the parameters of misalignement correction and beam hardening correction were chosen between 0 and 1.5 and between 51\% and 56\%, respectively. Each reconstructed image has a size of $1024 \times 1024$ pixels and a resolution of $81 \mu \mathrm{m}$. The reconstructed image stack was first inspected with Paraview and Fiji, all the successive analysis on the tomographic images were performed in MATLAB R2018b. In a tomographic image, the pores will appear in black (as in Fig. 1 , central columns).

Six 3-D numerical controls containing a random distribution of pores were also simulated. The goal was to take into account the great variety of pottery building techniques that could possibly show random pore patterns, such as moulding or pinching (see for instance [Roux 2016]) in contrast to the coiling technique and SPT. These numerical controls were simulated by randomly disposing into 3-D volumes a number of pores that is comparable to the average number of pores in a real sherd, once the latter are counted inside archaeological and experimental sherds through the segmentation protocol. These virtual volumes are either boxes, whose size is comparable to the archaeological sherds' size (i.e., $6 \times 6 \times 2 \mathrm{~cm}^{3}$ maximum) or cylinder portions ( $5 \mathrm{~cm}$ diameter and $2 \mathrm{~cm}$ thickness, as for experimental controls). The size 
of the voxel is the same as the one used for the sherds acquired through $\mu$-CT (81 $\mu \mathrm{m})$. These numerical controls are named as $N u m X X$, where $X X$ represents a couple of digits from 01 to 06 .

\subsection{Pore segmentation}

To the Authors' knowledge, there is no standard protocol for the analysis of the complex and heterogeneous tomographic images acquired on ancient pottery. The first objective of the analysis being the extraction of pore regions from the 3-D datasets on large ROIs, a new protocol was developed and adapted to the available data set. All consecutive steps of this protocol were performed on MATLAB R2018b, with both new code and standard MATLAB routines, which are specified in the following. Each step is illustrated with an example in Fig. 2. The whole volume of the sherd for the considered example is displayed in Fig. 2a.

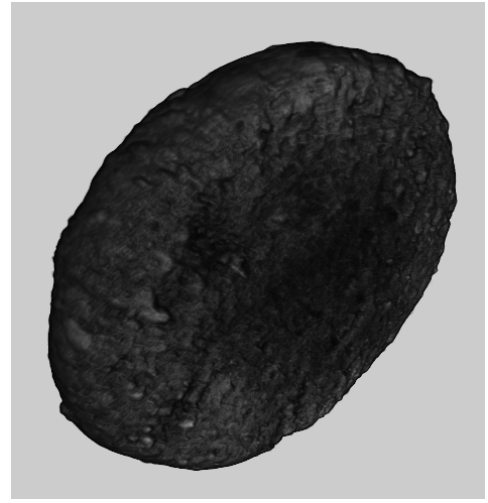

(a)

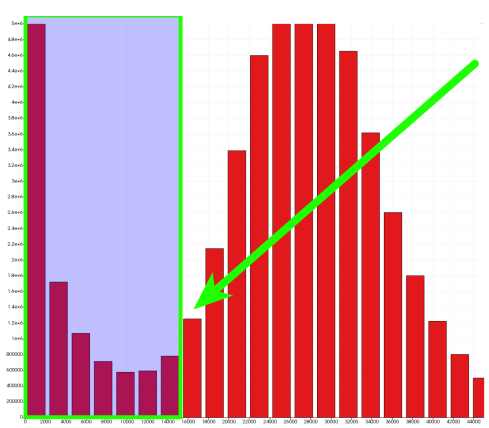

(b)

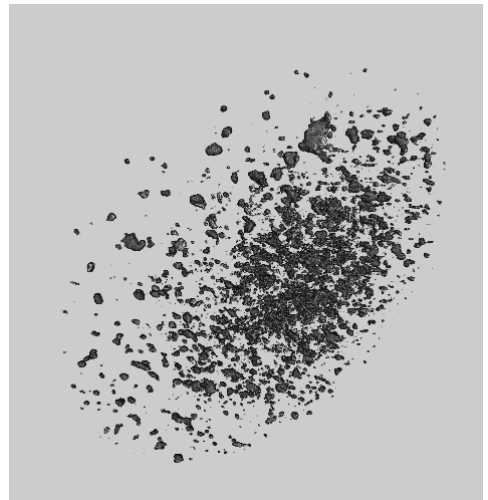

(c)

Figure 2: Pore segmentation process. (a) SPT control (SP1, $5 \mathrm{~cm} \varnothing, 1 \mathrm{~cm}$ thickness, approximately), volume. (b) Voxels' lower intensities, corresponding to pores (Step I). (c) Final segmentation result for SP1.

Step I (Voxel identification). The preliminary step is the identification of the voxels containing the air, characterized by low X-Ray absorption: to this aim, the distribution of the greyscale intensity values is evaluated in the whole sherd volume and the voxels with low intensities, i.e. low absorption, are selected by the user (Fig. 2b). The choice of the cutting threshold depends on the histogram's values and it is decided by the user for each dataset. This procedure allows to select the voxels corresponding to air location (which have intensity equal to 0 ) as well as the voxels which are located on and near the boundary of the regions which contour the pores inside the sherd: as the pores contours are not sharp due to tomographic resolution, 
these voxels are selected as pores to avoid information loss on pore thickness.

Step II (Volume binarisation). A binary volume is obtained,

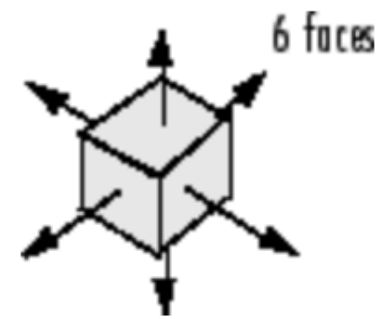

Figure 3: 6connectivity scheme for connected component identification (from MATLAB online documentation).

where the air (including the air surrounding the ceramic sample, which will be referred to as background, and the porosity inside the sample) is separated from other materials contained in the pottery (as in Fig. 4 second column, the white value is assigned to air voxels and the black value is assigned to the materials). The MATLAB function imbinarize ${ }^{1}$ is used in this step.

Step III (Connected components detection). A method for the detection of the connected components of the air voxels is applied, in order to identify the largest connected component which corresponds to the background and remove it (as in Fig. 2c). The MATLAB function bwconncomp ${ }^{2}$ is used with the parameter conn set to 6 , which returns the connected components in binary images with a connectivity of 6 for 3-dimensional images (i.e., voxels are connected if they share a common face among the 6 possible faces, as in Fig. 3). The 6-connectivity was chosen as it allows to keep the pores' voxels as separated as possible.

An example of the connected component detection process is shown in Figure 4 for a 2-D slice issued from a coiled experimental control volume and for a 2-D slice issued from a SPT experimental control volume. After the voxel selection implemented in Step I, the 2-D binary slide is obtained in Step II as shown in Fig. 4, second column. In Step III, the connected components in the images are retrieved in the binary volume and labeled (in colours in Fig. 4, third column). The pixels corresponding to the background region are detected and set to black, so that only the position associated to internal pores remains in the data (as in Fig. 4, right column). The minimal size of the remaining pores is 1 voxel. It is important to note that it unachievable to automatically discriminate small components which are due to noise from actual pores. Noise effect reduction is applied after the evaluation of the Hough transform.

\footnotetext{
${ }^{1}$ https://www . mathworks.com/help/images/ref/imbinarize.html

2https://www.mathworks.com/help/images/ref/bwconncomp.html
} 


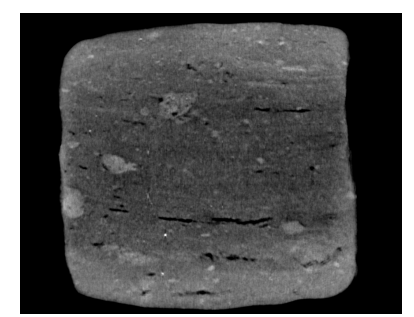

(a)

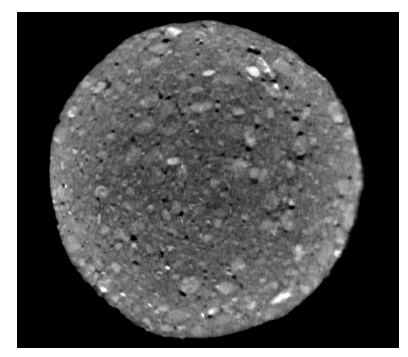

(e)

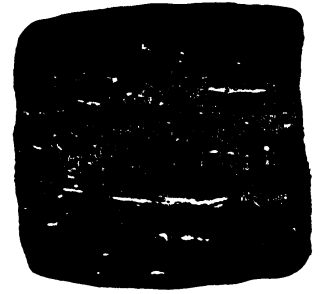

(b)

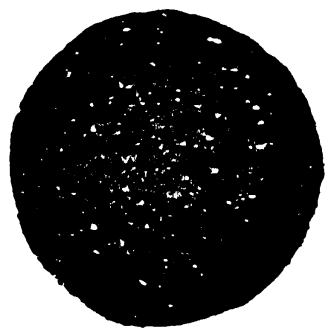

(f)

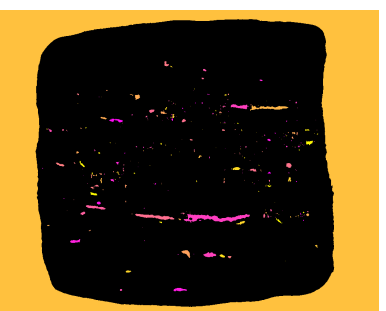

(c)

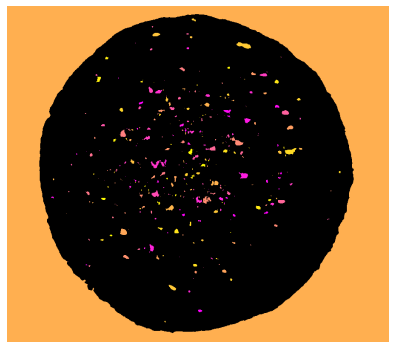

(g)

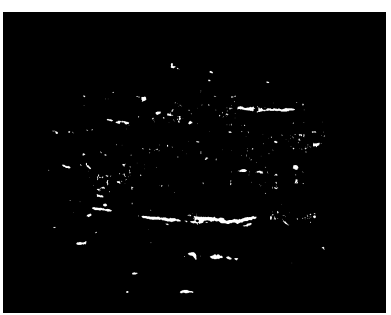

(d)

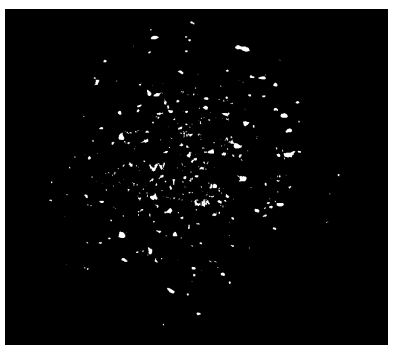

(h)

Figure 4: Connected components detection process (2-D examples). First row: central slice from coiling control C1, $6 \mathrm{~cm}$ side approximately, second row: central slice from SPT control SP1, $5 \mathrm{~cm} \varnothing$ approximately. (a) and (e): slice; (b) and (f): binary image (Step II); (c) and (g): region labels in colours (Step III); (d) and (h): final results.

\subsection{Hough transform}

Once pore locations are identified inside the volume, the successive goal was detecting the principal distribution features of pores inside the sherds in order to diagnose the building technique.

To this aim, the Hough transform is exploited, which is a classical tool in Image Processing for the automatic detection of points distributed along straight lines or parametric shapes in images [Ballard 1981, Beltrametti et al. 2013, Duda and Hart 1972, Gonzalez and Woods 2006, Hart 2009, Hough 1962, Mukhopadhyay and Chaudhuri 2015, Tu 2014], and is related to the integral Radon transform [vanGinkel et al. 2004]. Other methods, like PCA based object recognition methods or other template matching methods are appropriate for shape detection in images. In the present issue, however, these shapes (parallel lines or circles) are not directly present in the available images, so the detection can only be performed on points or small regions (pores) that are located along them. Thus, these techniques are not suitable for this problem, while the Hough transform is appropriate. The Hough transform relates points from an image space to a voting array in an associated parameter space (called Hough space). Thus, it is possible to setup a shape detection issue as a peak detection process which involves vote counting in a discretised accumulator space. 
In 2-D, the classical Hough transform processing associates points in the image space to sinusoids in the Hough space, so that the line detection issue is transformed in the problem of finding and counting intersection points between these sinusoids. The parallelism between lines corresponds to the superposition (same abscissa) of intersection points in the Hough space. The Hough transform relies on the so-called normal parametrisation of lines and on a voting procedure in a parameter space [Duda and Hart 1972]. Two Cartesian planes are considered, the space $x O y$ which corresponds to the image space, and the associated parameter space $\theta O \rho$, which is called Hough space (see Fig. 5). The normal parametrisation defines a line $\ell$ in the image space $x \mathrm{Oy}$ by means of two parameters, the distance $\rho$ between the line $\ell$ and the origin and the angle $\theta$ formed by the horizontal axis $0 x$ and the normal vector to $\ell$ (see Fig. 5a):

$$
(x, y) \in \ell \Longleftrightarrow x \cos \theta+y \sin \theta=\rho
$$

The couple $(\theta, \rho)$ which identifies the line (1) in the image space is transformed into a point $(\theta, \rho)$ in the Hough space. The Hough space is generally restricted to $\rho \in[0, M]$ where the constant $0<M<+\infty$ relates to the image space dimensions and $\theta \in[0, \pi]$ to avoid redundancy in line identification. Given a point $P=(x, y)$ in the image space, all the lines which pass through $P$ are defined via (1) by a set of parameters $(\theta, \rho)$, which forms a sinusoidal curve in the Hough space (for example, the red point in the image space of Fig. 5b is transformed into the red sinusoid in the Hough space of Fig. 5c). Conversely, each point $(\theta, \rho)$ in the Hough space identifies a line via (1) in the image space (for example, the green point in Fig. 5c identifies the green line in Fig. 5b). The Hough space is used as a discrete accumulator space, where each point $(\theta, \rho)$ which lies on a sinusoid counts a vote. For example, in Fig. 5c the points on the red sinusoid which are not intersection points for others sinusoids count 1 vote each, while the green point counts 2 votes, as it is the intersection point of 2 sinusoids (which correspond to 2 points aligned in the image space of Fig. 5b). If $N$ points are on the same line $\ell^{N}$ in the image space, the line $\ell^{N}$ is identified by a point $\left(\theta^{N}, \rho^{N}\right)$ which is the intersection point of $N$ sinusoids in the Hough space (for example, the orange point in Fig. 5c corresponds to the orange line which passes through 3 points in the image space in Fig. 5b). In conclusion, the search for points which are aligned in the image space can be carried out by the 


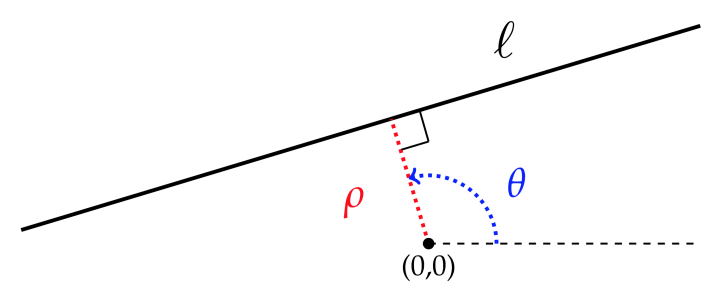

(a) Normal parametrisation of lines

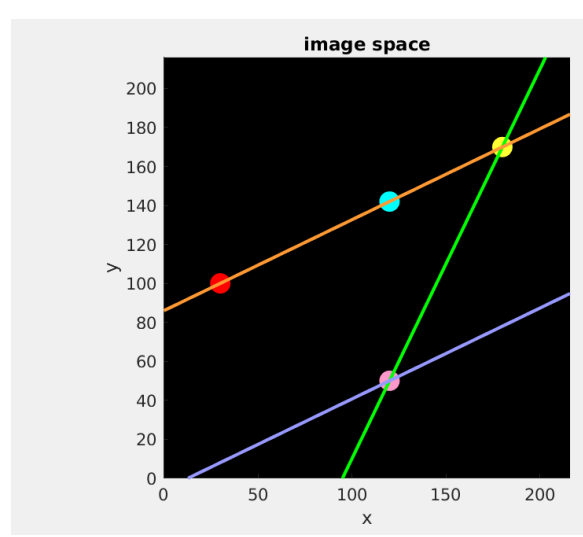

(b) Image space

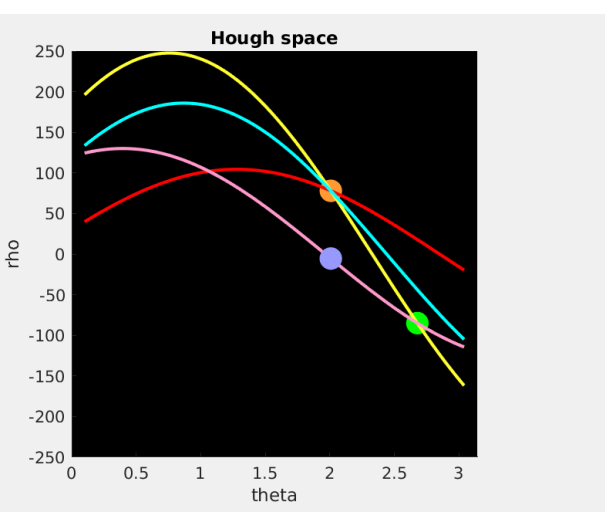

(c) Hough space

Figure 5: Fig (a): Normal parametrisation of lines. From (b) to (c): Example of Hough transform processing (bi-dimensional case). Red, cyan, pink and yellow points in image space (left) transform to sinusoids in Hough space (right); green, violet and orange points in Hough space (right) correspond to lines in the image space (left).

search for points that count the greater amount of intersection between sinusoids in the Hough space, whence of votes in the discrete accumulator space. Furthermore, points on parallel lines in the image space correspond to groups ( 1 group per line) of sinusoids that intersect at the same value of $\theta$ in the Hough space.

This approach can be generalised to the detection of other shapes, like circles, parabolas and other parametric curves [Ballard 1981, Beltrametti et al. 2013].

Depending on the object under investigation, the Hough transform procedure can be adapted to the detection of shapes in the 3-D space [Torrente et al. 2018]. A numerically efficient way to detect lines in 3-D point clouds is described in [Dalitz et al. 2017] (with $\mathrm{C}++\operatorname{code}^{3}$ ). This 3-D Hough transform is used to determine alignment in pores inside the sherds. The ability of the Hough transform to discriminate between pore location distributions on parallel straight lines from other distributions is particularly efficient and convenient in the present situation, in order to distinguish the coiling technique from others.

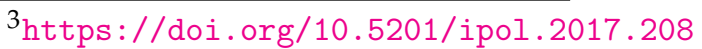




\section{Results and discussion}

The 3-D Hough transform is used to detect principal alignments in pores inside the datasets. Indeed, as already discussed in Section 1, macro- and microscopic analyses performed on archaeological and experimental pottery showed that parallel lines patterns are characteristic of the coiling technique, while other patterns are likely to be related to other building techniques. The detection of circular patterns via the Circular Hough transform [Atherton and Kerbyson 1999, Duda and Hart 1972] is preliminary investigated as well.

\subsection{Line detection}

After implementing the segmentation process described in Section 2.2, the 3-D Hough transform was applied to the data volumes [dataset] which contain the pores' coordinates for each of the considered samples (ceramic and numerical), and the detected lines were calculated.

In the experiments, the 15 lines which obtained the most votes in the Hough space (i.e., the lines which pass through the greater amount of pores in the samples) are retained and the lines are displayed in the original image space. The choice of 15 lines appears to be an appropriate trade-off which allows to take into account the principal alignments of pores/voxels and remove some noise, discarding the lines which are related to a small count of votes (e.g., see the results in Fig. 6, right column).

Now, line parallelism has to be investigated. To this aim, for each dataset the direction (unit) vectors $\mathbf{b}_{i}, i \in\{1, \ldots, 15\}$ of the lines detected by the 3 -D Hough transform process are considered. If two lines $i$ and $j$ have almost the same direction, the scalar product $p_{i j}=\left\langle\mathbf{b}_{i}, \mathbf{b}_{j}\right\rangle$ of their direction vectors is almost equal to 1 , while it is close to 0 for almost orthogonal lines. Thus, for each dataset $105\left(=\left(\begin{array}{c}15 \\ 2\end{array}\right)=C_{15}^{2}\right)$ scalar products' values $p_{i j}$ are calculated. The statistical analysis of the scalar products' values though histograms and descriptive statistics (minimum and maximum values, median, $1^{\text {st }}$ and the $3^{\text {rd }}$ quartiles) allows to discriminate between the different fabrication techniques.

The data set considered for the analysis includes 22 elements:

- 4 sherds formed with the coiling technique (3 experimental controls and 1 archaeological sherd), 
- 12 sherds formed with SPT (7 experimental controls and 5 archaeological sherds)

- and 6 numerical controls with a random distribution of pores.

For each data set, pore segmentation is performed (e.g., see the results in Fig. 6, central columns), followed by Hough transform's line detection. A visual inspection of the Hough transform's results show that most of the detected lines are parallel in the case of the sherds built with the coiling technique (e.g., see Figs. 6a and 6b, right column), while the lines intersect more in the case of those built with SPT (e.g., see Figs. $6 c$ and $6 d$, right column) and the numerical controls (e.g., see Fig. 6e, right column). It is important to note that the lines for the SPT experimental control tend to intersect around the centre (see Fig. 6c, right column).

To strengthen the visual inspection, the 105 scalar products are evaluated for the 15 Hough detected lines for each dataset of each class. Fig. 7a presents the histograms for the 5 classes. As each class contains a different quantity of datasets, the number of occurrences of each scalar product value for any given class is rescaled by dividing it by the quantity of datasets in that class, in order to allow comparisons between different classes. The statistics on the scalar product values of each class are shown in Fig. 7b. In each box plot, the low and the high black bars correspond to the minimum and the maximum values of the scalar products for the considered dataset; the lowest and the highest blue bars of the whiskers correspond to the $1^{\text {st }}$ and the $3^{\text {rd }}$ quartiles and the red bar corresponds to the median value.

The results show that a greater occurrence of almost parallel lines (scalar products close or equal to 1) is found in the cases of the coiling sherds, while for the SPT sherds and the numerical controls the scalar products span a greater range of values, corresponding to lines with different directions.

The scalar products of the Hough transform's results provide an accurate discriminating feature which allows to separate the coiled sherds from the SPT sherds and from the numerical controls: the high number of parallel lines detected by the 3-D Hough transform. An important remark is that the statistics for archaeological and experimental datasets are quite similar for both techniques which enforces the fact that the features are well appropriate. In addition to classification, this approach allows to detect the lines in the sherds for further analysis and modelling of the coiling technique.

In order to define characterising features for the SPT, circle detection is considered. 

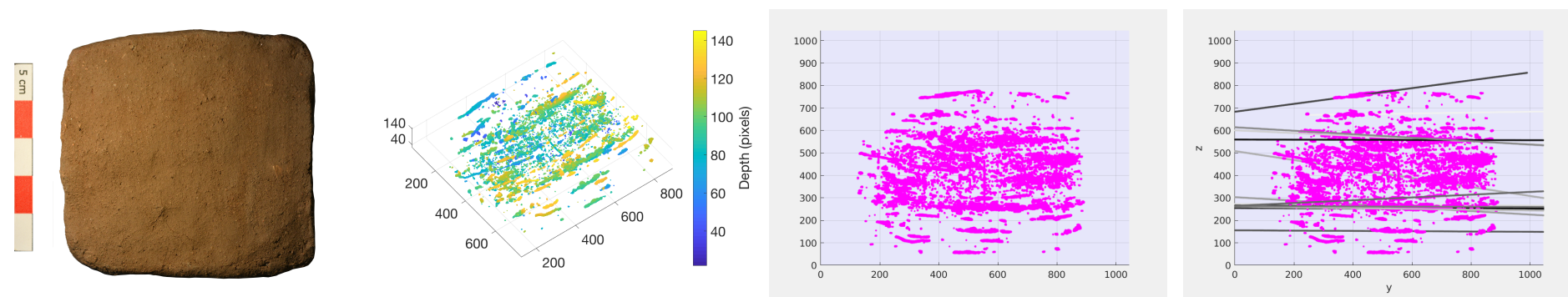

(a) Coiling technique, experimental sherd (C1)
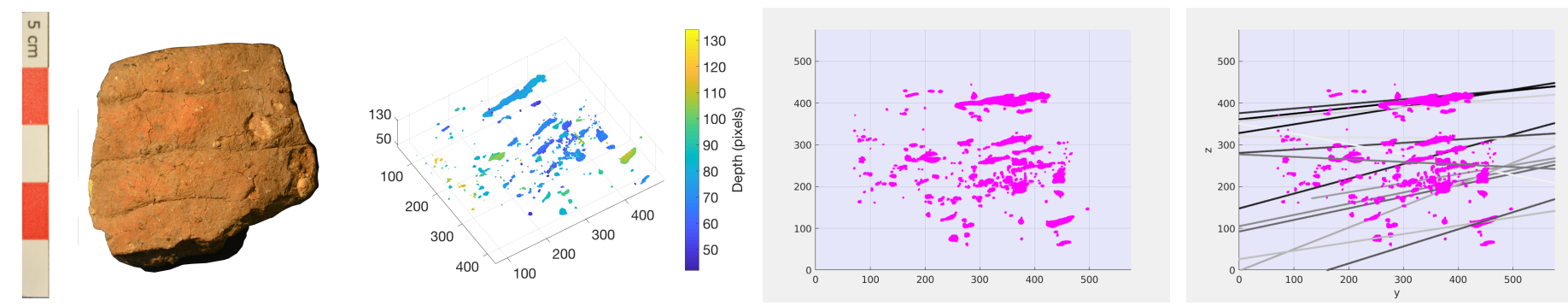

(b) Coiling technique, archaeological sherd (AC1)
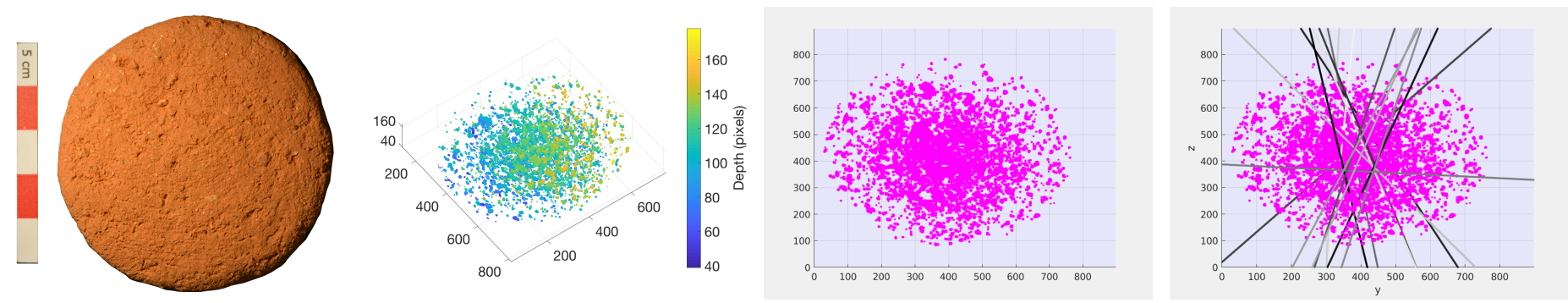

(c) SPT, experimental sherd (SP1)
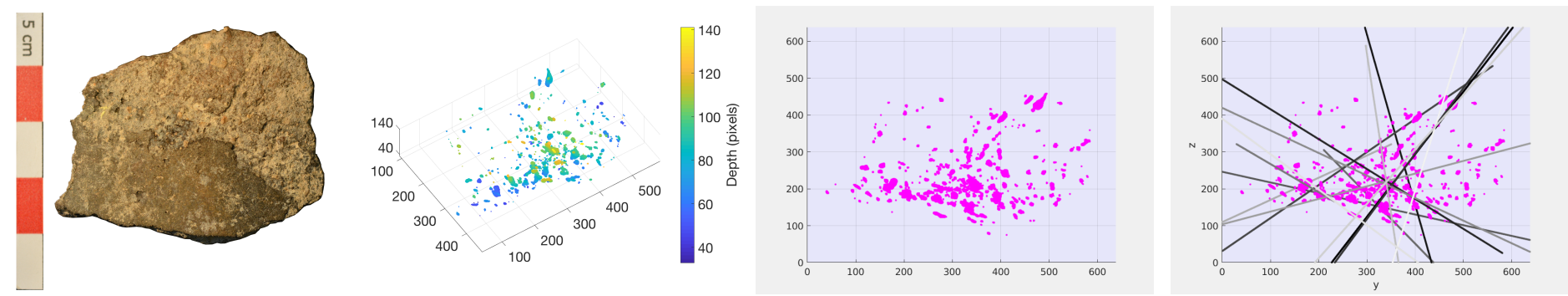

(d) SPT, archaeological sherd (ASP1)
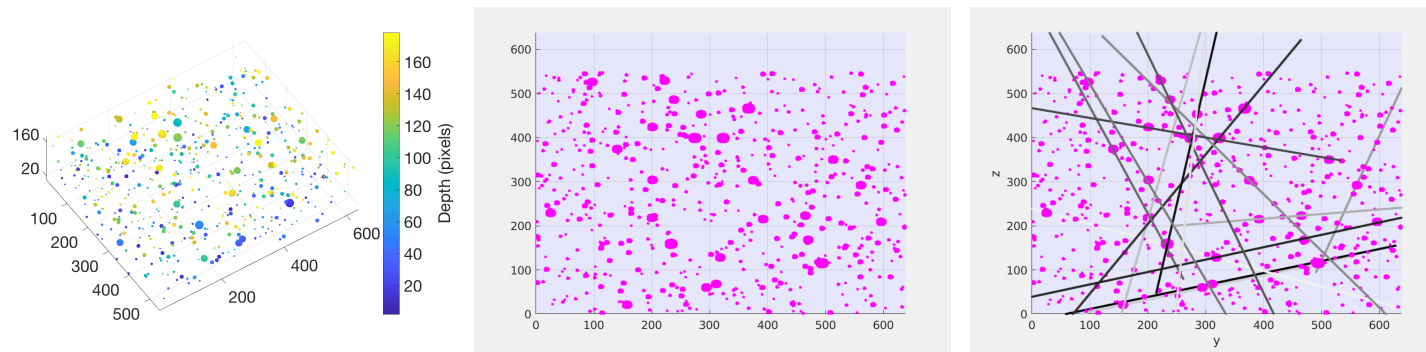

(e) Numerical control (Num01)

Figure 6: Left column: pottery sherds. Central columns: segmentation results showing the spatial distribution of pores. In the 3-D visualisation (second column), blue tones indicate the pores which are close to the internal surface of the sherd, while yellow tones indicate the pores which are close to the external surface of the sherd, respectively. In the 2-D visualisation (third column), a projection of 3-D pores' regions onto the tangential plane is shown. Right column: 3-D Hough results. Pores' voxels are displayed in pink colour and the detected lines are displayed in greyscale with decreasing intensity from black to white following the decreasing order of vote counts. 


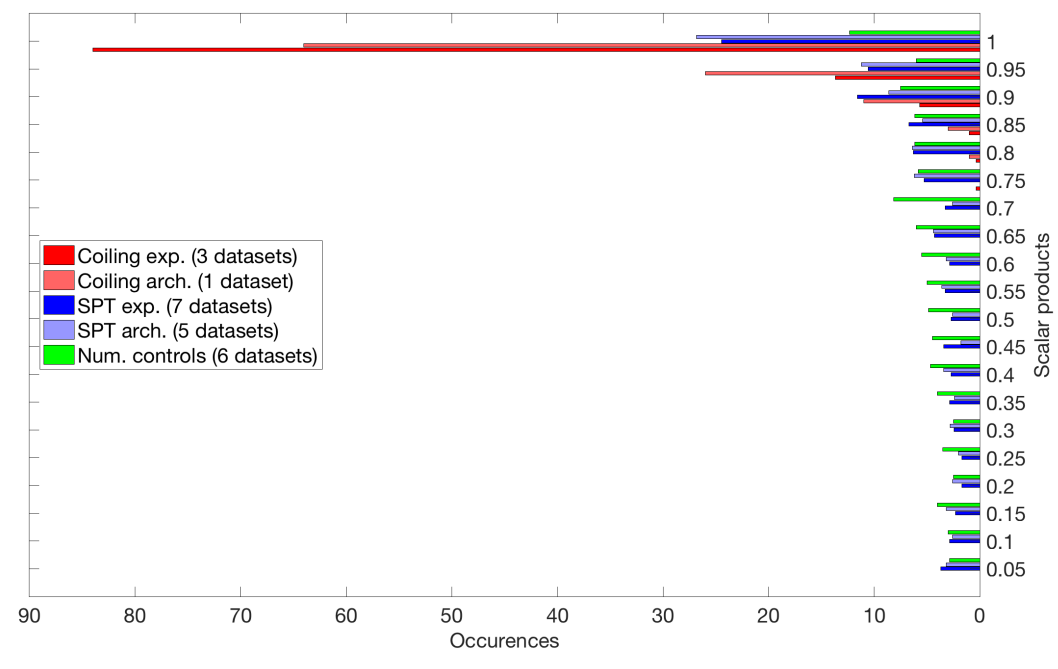

(a)

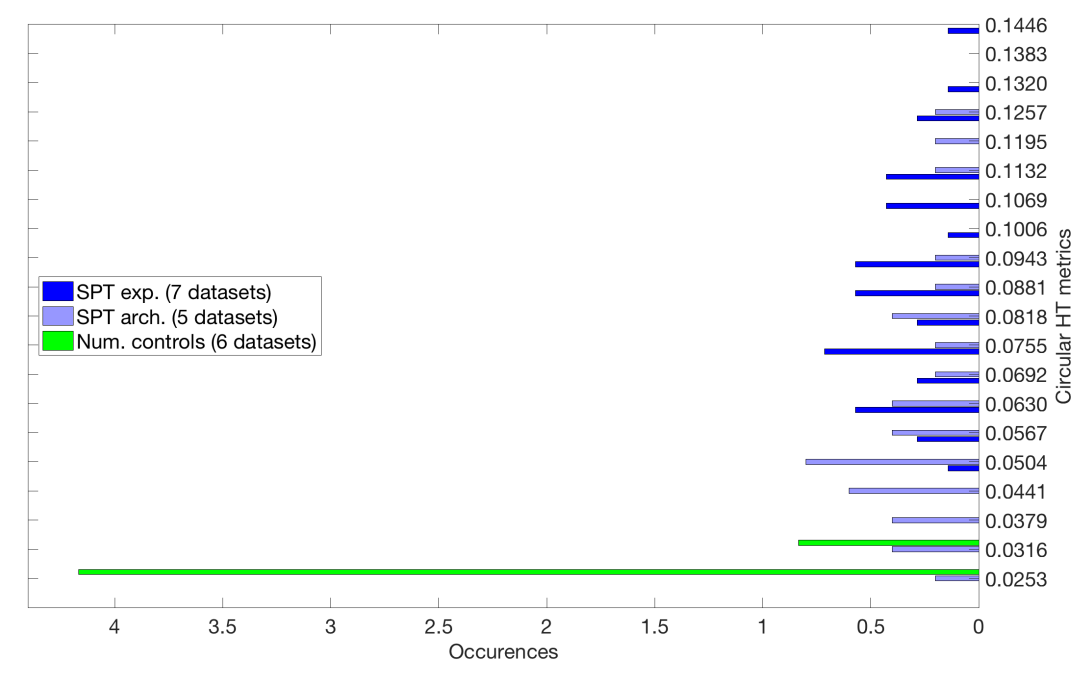

(c)

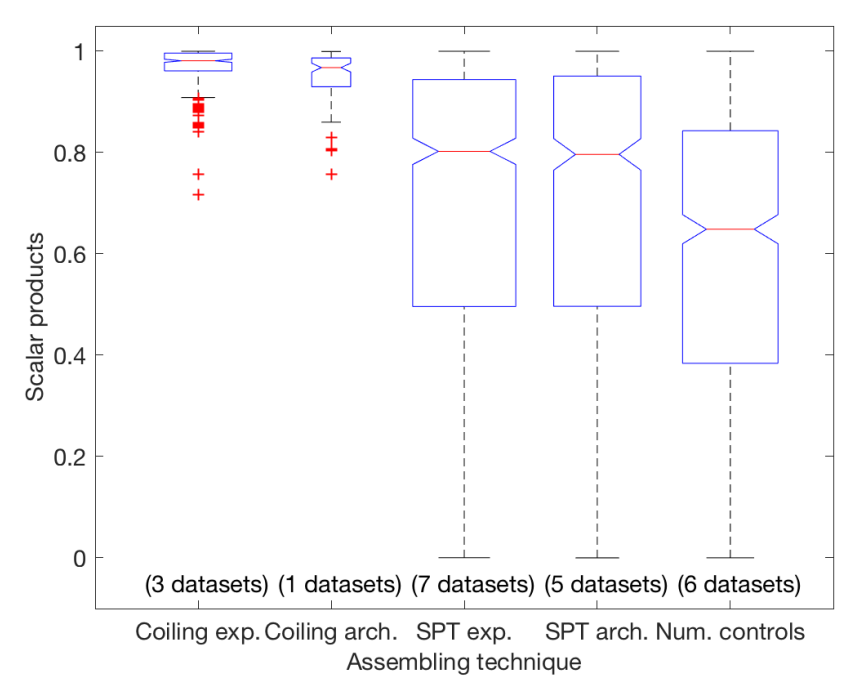

(b)

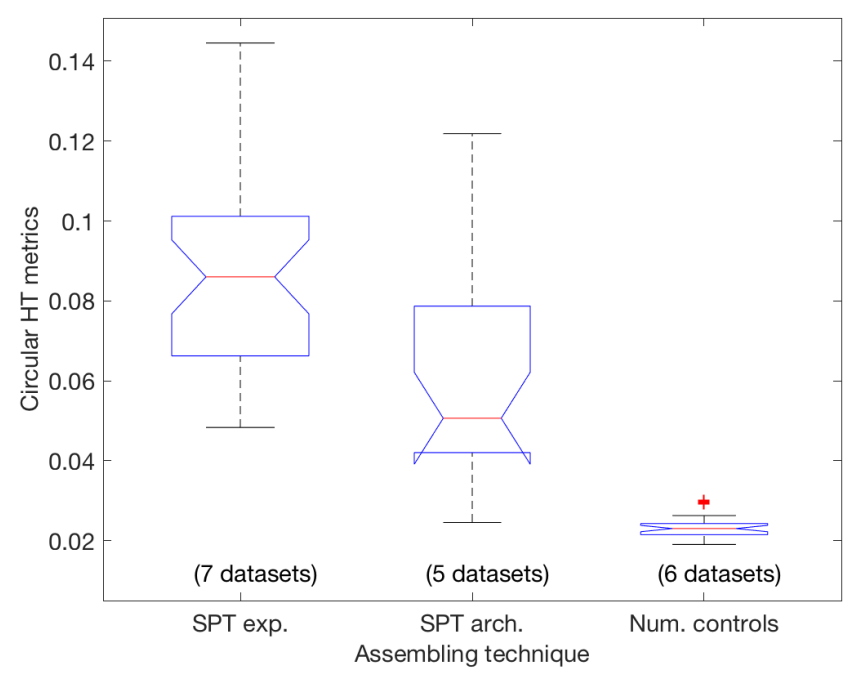

(d)

Figure 7: Statistics on results. In the histograms, the red colour corresponds to coiled sherds, the blue colour to the SPT sherds and the green colour to the numerical control in the histograms plots. In each box plot, the low and the high black bars correspond to the minimum and the maximum values of the scalar products for the considered dataset; the lowest and the highest blue bars of the whiskers correspond to the $1^{\text {st }}$ and the $3^{\text {rd }}$ quartiles and the red bar corresponds to the median value. The width of each box plot is proportional to the quantity of sherds in the related class. From (a) to (b): Histogram and box plot for scalar products of 15 lines for 16 sherds ( 3 experimental controls and 1 archaeological sherd for the coiling technique, 7 experimental controls and 5 archaeological sherds for the SPT) and 6 numerical controls. From (c) to (d): Histogram and box plot for the Circular Hough transform's metrics of 5 circles for 12 sherds (7 experimental controls and 5 archaeological sherds for the SPT) and 6 numerical controls. 
The Hough transform approach is already extended to a large variety of curves (including circles) with algorithms and available codes. As the SPT sherds are expected to show circular/spiralling patterns regarding pore disposition, while no such pattern should appear in the case of the numerical controls in which the pores are randomly disposed, an exploratory analysis was conducted to evaluate whether it was possible to distinguish their respective pore systems using the circular Hough transform.

\subsection{Preliminary results on circles detection}

In this section, preliminary investigations are described for the detection of circular patterns in the pores' disposition, in order to find distinctive features for the SPT building technique.

If the 3-D Hough transform for line detection is already able to provide quite different and discriminating results between the coiled sherds from the SPT ones, it does not really allow to distinguish between SPT sherds and numerical controls (see above Section 3.1). As the final goal is to detect quantifiable features which could characterise the SPT within the great variety of pottery building techniques, a generalisation of the Hough transform is applied, focusing the experiments on the twelve SPT sherds and the six numerical controls (described in Section 2.1).

To simplify the preliminary analysis, the Circular Hough transform [Atherton and Kerbyson 1999, Duda and Hart 1972] was tested for the detection of circles in 2-D images, by means of the the function imfindcircles ${ }^{4}$ available in MATLAB R2018b. The 2-D images considered for these experiments were constructed as follows: for each 3-D datasets of pores, all pores' points are projected onto a plane which is tangential to the largest face of the considered sample (e.g., see Fig. 8, left column). These images were fed to the function imfindcircles, which returns the center, radius and metric of the detected circles; the parameter metric contains a value which is proportional to the votes obtained by a circle (i.e., an high metric for a given circle corresponds to an high number of points on that circle). This process is actually similar to the one described for line detection in Section 2.3, except that it uses the classical parametrisation of circles, and it is led to detect peaks within intersection points between circles in the corresponding 3-D Hough space. For the experiments, the 5 circles which

\footnotetext{
${ }^{4}$ https://www . mathworks.com/help/images/ref/imfindcircles.html
} 
obtained the highest metrics between the circles with radii in the range $[50,300]$ pixels (corresponding to approximately $[50,300] \mathrm{mm}$ in real world measures) were selected.

A visual inspection of the circles detection results via the Circular Hough transform only allows to notice little differences between the SPT datasets and the numerical controls ones: the detected circles appears to be closer in the SPT case, while they are more scattered in the case of the numerical controls (e.g., see Fig. 8, right column). The metric results for all the twelve SPT datasets (7 experimental controls and 5 archaeological sherds) and six numerical controls datasets are shown in Fig. 7c. For each dataset the 5 metric values which are associated to the 5 detected circles are taken into account. Again, the results are grouped by dividing them into 3 different classes (SPT experimental controls, SPT archaeological sherds and numerical controls). As each class contains a different quantity of datasets, the number of occurrences of each scalar product value for any given class is rescaled by being divided by the quantity of datasets in the class, in order to allow comparisons between different classes. The statistics on the metric values of each class are shown in Fig. $7 \mathrm{~d}$.

The results show that the metric values for the SPT datasets are always higher than those of the numerical controls datasets: this indicates that the detected circles always pass through a greater number of points (i.e., pores) in the case of the SPT sherds, while the detected circles meet less points in the numerical controls cases. These differences on 2-D images preliminary show the possibility to characterise the SPT building technique with the Circular Hough transform and open the path to further development and analysis of 3-D approaches.

\section{Conclusion}

Within the framework of this research, a micro-tomographic analysis protocol is advocated, which is primarily based on the exhaustive visual analysis of the archaeological corpus and on the production of experimental controls shaped under controlled conditions with sourced geomaterials. It is therefore thanks to a thorough knowledge of the archaeological corpus that choices were made in terms of sampling regarding micro-tomographic analyses and 3-D data processing, allowing for proposing a new quantitative protocol for the characterisation of ancient pot- 

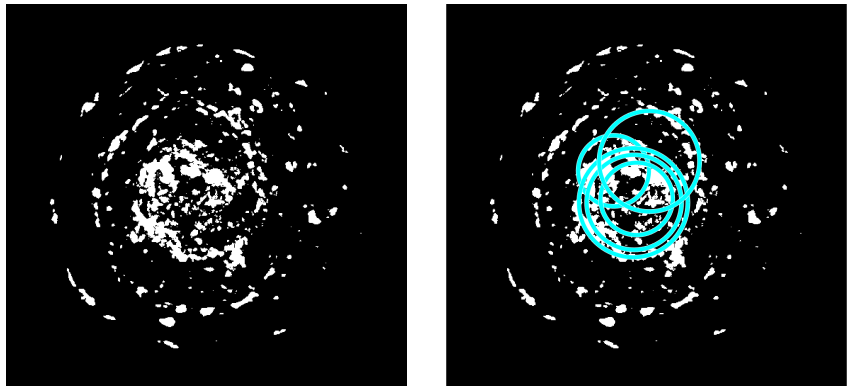

(a) SPT, experimental sherd (SP3)
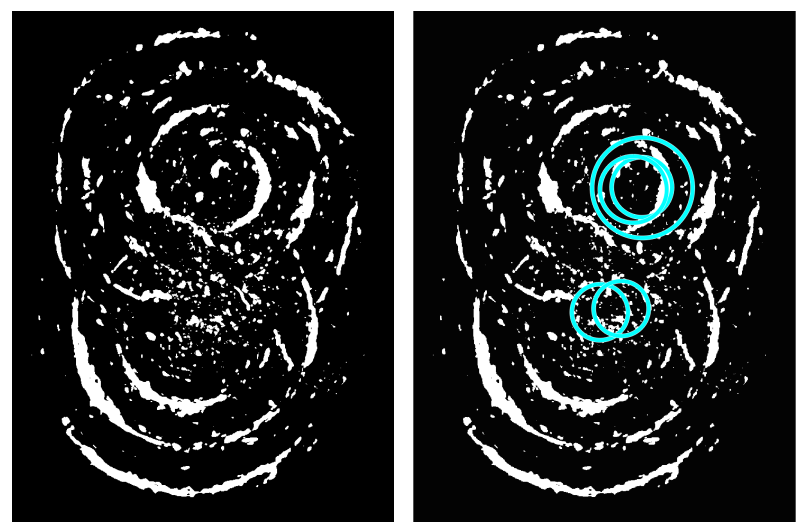

(b) SPT, experimental sherd (SP2)
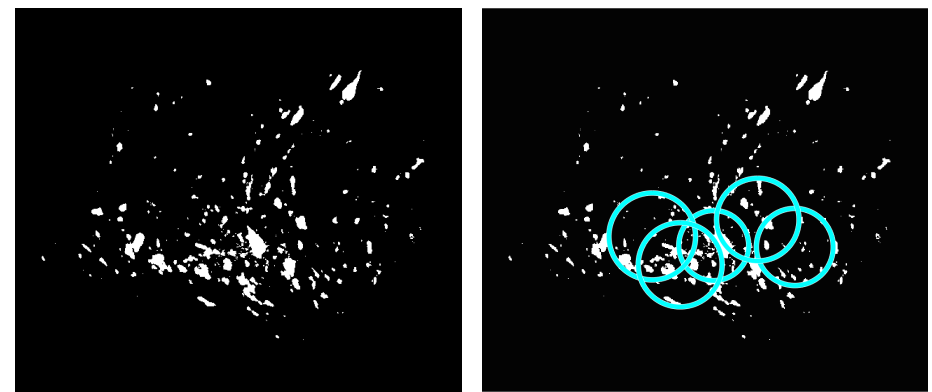

(c) SPT, archaeological sherd (ASP1)
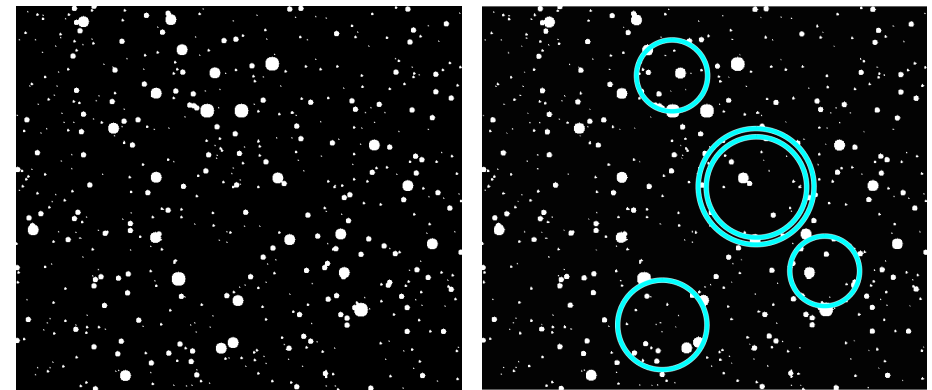

(d) Numerical control (Num01)
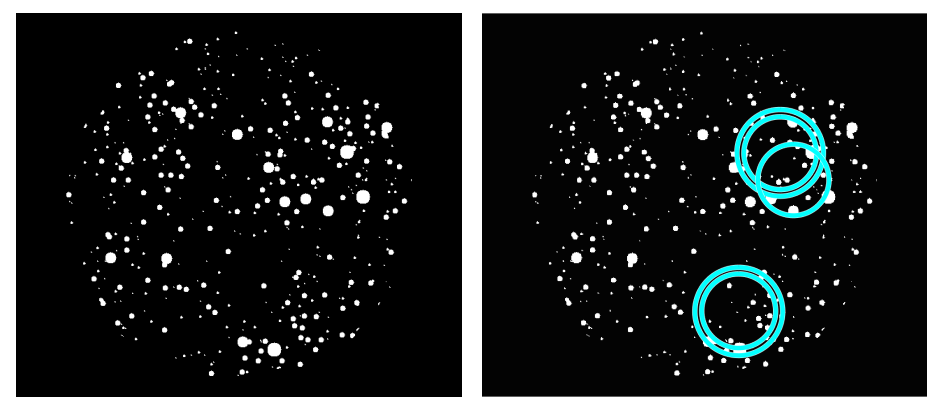

(e) Numerical control (Num04)

Figure 8: Circular Hough transform results. Left column: the 2-D projection of 3-D pores' disposition. Right column: the Circular Hough transform results. 
tery techniques. This analysis represents the first attempt for the quantitative characterisation of Neolithic pottery forming techniques using 3-D datasets and large ROIs. The 22 analysed datasets (16 archaeological and experimental sherds, as well as 6 numerical controls) provide a significant amount of statistically representative quantitative data that (1) strongly support the definition of the two ceramic techniques identified in the considered archaeological contexts and (2) provide a solid and reproducible frame of reference for the characterisation of the SPT technique, a pottery technique for which there are no points of comparison among archaeological or actualist contexts.

Hough transform tools appear extremely promising for the characterisation and classification of the internal architecture of both archaeological and experimental samples. The implemented 3-D Hough transform protocol allowed for effectively discriminating the porous systems respectively associated with the coiling technique and the SPT, by comparing the distributions of the scalar products between the directions of the recovered lines: these recovered lines are mainly parallel for coiling sherds, while they intersect for the SPT sherds. The advantage of analysing these different classes of samples via the detection of a same geometrical shape (line) permitted to overcome the diversity of macrotraces observed on the archaeological and experimental sherds and to build a reference frame independent from the visual analysis of the sherds. Moreover, the possibility to relocate the detected shapes inside the sherds could allow further analysis on the pores, i.e. their size or distribution inside the sherd. Ongoing work is also dedicated to setting up a Deep Learning classification framework which could be applied to tomographic pottery images.

The use of the 2-D Circular Hough transform then permitted to distinguish the porous systems associated with the SPT sherds from random porous systems. This important step, which will be developed in 3-D on circle and spiral shapes as part of future investigations, is an essential milestone in the characterisation of SPT. Moreover, this approach will be applied to a larger number of samples, notably including archaeological and experimental samples formed using other ceramic forming techniques such as modelling, slab building, moulding or pinching. Furthermore, a future goal will be the detection of 2-D structures in available 3-D images, such as portions of planes or cylinders, as they may provide valuable information to distinguish the internal architectures associated with different ceramic forming techniques. Further research will also involve high resolution tomography data issued from Syn- 
chrotron facilities, in order to investigate and detect different types of pores, as some of them may not directly reflect the forming processes but the paste processing (i.e., addition of vegetal fibers).

\section{Data Availability Statement}

The data that support the findings of this study are available from the corresponding author upon reasonable request.

\section{Acknowledgements}

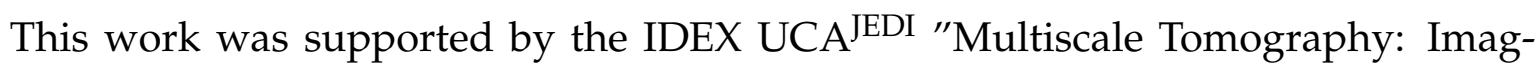
ing and Modelling Ancient Materials, Technical Traditions and Transfers - TOMAT" project and by the Agence Nationale de la Recherche "Western Mediterranean Impressed Wares. An interdisciplinary research on Early Neolithic pottery (6th millennium cal BCE): sourcing, production, uses and transfers" project.

The Authors thank the anonymous reviewers who provided helpful and constructive comments that contributed to improve the manuscript.

VLC thanks M. Gabriele (GEOAZUR, CEPAM - UMR 7264) and G. Durrenmath (CEPAM - UMR 7264) for their precious help and the valuable discussions.

VLC, LBF, JL, LG and DB designed the project and wrote the paper. VLC developed the mathematical method with support from LBF, JL and SC. LG and DB performed the technological analysis of the archaeological corpus and produced the experimental controls. DP acquired the tomographic data. All authors reviewed the manuscript and discussed the results.

\section{References}

[Atherton and Kerbyson 1999] T.J. Atherton and D.J. Kerbyson. Size invariant circle detection. Image and Vision Computing, 17(11):795-803, 1999.

[Ballard 1981] D.H. Ballard. Generalizing the Hough transform to detect arbitrary shapes. Pattern Recognition, 13(2):111-22, 1981. 
[Beltrametti et al. 2013] M. Beltrametti, A. Massone and M. Piana. Hough Transform of Special Classes of Curves. SIAM Journal on Imaging Sciences, 6(1):391-412, 2013.

[Binder et al. 1993] D. Binder, E.J. Brochier, H. Duday, D. Helmer, P. Marinval, S. Thiébault and J. Wattez. L'abri Pendimoun à Castellar (Alpes-Maritimes). Nouvelles données sur le complexe culturel de la céramique imprimée méditerranéenne dans son contexte stratigraphique. Gallia Préhistoire, 35:177251, 1993.

[Binder et al. 2020] D. Binder, J. Battentier, L. Bouby, J.-E. Brochier, A. Carré, T. Cucchi, C. Delhon, C. De Stefanis, L. Drieu, A. Evin, L. Girdland Flink, G. Goude, L. Gourichon, S. Guillon, C. Hamon and S. Thiébault. First farming in the northwestern Mediterranean: evidence from Castellar - Pendimoun during the sixth millennium BCE, in Gron, K.J., Rowley-Conwy, P., Sorensen, L. (Eds.), Farmers at the Frontier: A Pan-European Perspective on Neolithisation: 145-59. Oxbow, Oxford \& Philadelphia, 2020.

[Coletti et al. 2016] C. Coletti, G. Cultrone, L. Maritan and C. Mazzoli. Combined multi-analytical approach for study of pore system in bricks: How much porosity is there? Materials Characterization, 121:82-92, 2016.

[Commenge 2009] C. Commenge. Techniques séquentielles appliquées aux argiles: résonances orientales dans le Néolithique de Macédoine, pages 205-20. Archives d'Écologie Préhistorique, Toulouse, 2009.

[Dalitz et al. 2017] C. Dalitz, T. Schramke and M. Jeltsch. Iterative Hough Transform for Line Detection in 3D Point Clouds. SIAM Journal on Imaging Sciences, 7:18496, 2017.

[dataset] The data that support the findings of this study are available from the corresponding author upon reasonable request.

[Drieu et al. 2019] L. Drieu, M. Horgnies, D. Binder, P. Pétrequin, A.-M. Pétrequin, K. Peche-Quilichini, T. Lachenal, M. Regert. Influence of porosity on lipid preservation in the wall of archaeological pottery. Archaeometry, 61(5):1081-96, 2019.

[Drieu et al. 2020] L. Drieu, A. Lucquin, L. Cassard, S. Sorin, O.E. Craig, D. Binder and M. Regert. A Neolithic without dairy? Chemical evidence from the content 
of ceramics from the Pendimoun rock-shelter (Castellar, France, 5750-5150 BCE). Journal of Archaeological Science: Reports, 2020 (in press).

[Duda and Hart 1972] R.O. Duda and P.E. Hart. Use of the Hough Transformation to Detect Lines and Curves in Pictures. Commun. ACM, 15(1):11-15, 1972.

[Gomart 2010] L. Gomart. Variabilité technique des vases du Rubané récent du Bassin parisien (RRBP) et du Villeneuve-Saint-Germain (VSG) : un cas d'étude dans la vallée de l'Aisne. Bulletin de la Société préhistorique française, 107(3):53748, 2010.

[Gomart 2014] L. Gomart. Traditions techniques et production céramique au Néolithique ancien. Étude de huit sites rubanés du Nord-Est de la France et de Belgique. Leiden: Sidestone, 2014.

[Gomart et al. 2017] L. Gomart, A. Weiner, M. Gabriele, G. Durrenmath, S. Sorin, L. Angeli, M. Colombo, C. Fabbri, R. Maggi, C. Panelli, D.F. Pisani, G. Radi, C. Tozzi, D. Binder. Spiralled patchwork in pottery manufacture and the introduction of farming to Southern Europe. Antiquity, 91(360):1501-14, 2017.

[Gonzalez and Woods 2006] R.C. Gonzalez and R.E. Woods. Digital image processing. Prentice Hall, Upper Saddle River, N.J., 2008.

[Hart 2009] P. E. Hart. How the Hough transform was invented [DSP History]. IEEE Signal Processing Magazine, 26(6):18-22, 2009.

[Hough 1962] P. C. Hough. Method and means for recognizing complex patterns, U.S. Patent 3069654. December 1962, http://www.freepatentsonline. com/3069654.html

[Kahl and Ramminger 2012] W.-A. Kahl and B. Ramminger. Non-destructive fabric analysis of prehistoric pottery using high-resolution X-ray microtomography: a pilot study on the late Mesolithic to Neolithic site Hamburg-Boberg. Journal of Archaeological Science, 39(7):2206-19, 2012.

[Kozatsas et al. 2018] J. Kozatsas, K. Kotsakis, D. Sagris and K. David. Inside out: Assessing pottery forming techniques with micro-CT scanning. An example from Middle Neolithic Thessaly. Journal of Archaeological Science, 100:102-19, 2018. 
[Kreiter et al. 2017] A. Kreiter, T. Marton, L. Gomart, K. Oross and P. Pánczél. Looking into houses: analysis of LBK ceramic technological change on a household level. Matières à Penser: Raw materials acquisition and processing in Early Neolithic pottery productions, pages 111-32. Séances de la Societé préhistorique française, Paris, March 2017.

[Livingstone Smith 2001] A. Livingstone Smith. Pottery manufacturing processes: reconstruction and interpretation, in E. Garcea (ed.) Uan Tabu in the settlement history of the Libyan Sahara: 113-52. Florence: All'Insegna del Giglio, 2001.

[Mukhopadhyay and Chaudhuri 2015] P. Mukhopadhyay and B.B. Chaudhuri. A survey of Hough Transform. Pattern Recognition, 48(3):993-1010, 2015.

[Park et al. 2019] K.S. Park, R. Milke, E. Rybacki and S. Reinhold. Application of Image Analysis for the Identification of Prehistoric Ceramic Production Technologies in the North Caucasus (Russia, Bronze/Iron Age). Heritage, 2(3):2327-42, 2019.

[Pierret et al. 1996] A. Pierret, C.J. Moran and L.-M. Bresson. Application of Image Analysis for the Identification of Prehistoric Ceramic Production Technologies in the North Caucasus (Russia, Bronze/Iron Age). Journal of Archaeological Science, 23(3):419-28, 1996.

[Roux 2016] V. Roux. Ceramic manufacture: The chaîne opératoire approach. The Oxford handbook of archaeological ceramic analysis, pages 101-13. Oxford University Press, Paris, 2016.

[Salanova et al. 2010] L .Salanova, J. Vieugué and L. Gomart. Methods of study of large ceramic complexes: series and sets of Neolithic pottery from Kovachevo (Bulgaria). Arheologia Sofia, (3-4):7-23, 2010.

[Sanger et al. 2013] M. Sanger, J. Thostenson, M. Hill and H. Cain. Fibrous twists and turns: early ceramic technology revealed through computed tomography. Applied Physics A, 111(3):829-39, 2013.

[Sanger 2016] M. Sanger. Investigating pottery vessel manufacturing techniques using radiographic imaging and computed tomography: Studies from the Late Archaic American Southeast. Journal of Archaeological Science: Reports, 9:586-98, 2016. 
[Sanger 2017] M. Sanger. Coils, slabs, and molds: examining community affiliation between Late Archaic shell ring communities using radiographic imagery of pottery. Southeastern Archaeology, 36(2):95-109, 2017.

[Thér 2016] R. Thér. Identification of pottery-forming techniques using quantitative analysis of the orientation of inclusions and voids in thin sections. Archaeometry, 58:222-28, 2016.

[Torrente et al. 2018] M.-L. Torrente, S. Biasotti and B. Falcidieno. Recognition of feature curves on $3 \mathrm{D}$ shapes using an algebraic approach to Hough transforms. Pattern Recognition, 73:111-30, 2018.

[Tu 2014] C. Tu. Enhanced Hough transforms for image processing. PhD Thesis, Université Paris-Est, 2014. https ://tel. archives-ouvertes.fr/tel-01140138

[vanGinkel et al. 2004] M. van Ginkel, C.L. Luengo Hendriks and L.J. van Vliet. A short introduction to the Radon and Hough transforms and how they relate to each other. Number QI-2004-01 in the Quantitative Imaging Group Technical Report Series, pages 1-9, 2004. 